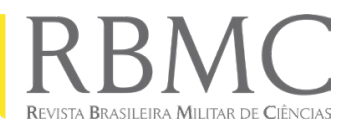

ISSN 2447-9071

doi https://doi.org/10.36414/rbmc.v6i16.71

Contato para correspondência: Vania Cristina Rodríguez Salazar

E-mail:

vaniaro2009@gmail.com

Conflito de interesse: Não

Financiamento: Recursos próprios

Recebido: $26 / 11 / 2020$

Aprovado: 03/12/2020

\section{Perfil sociodemográfico e padrão de uso de ecstasy por usuários residentes na cidade de Goiânia-GO}

\section{Sociodemographic profile and pattern of use of ecstasy by users resident in the city of Goiânia-GO}

\author{
Lívia Mendes de Freitas' ${ }^{1}$ Vania Cristina Rodríguez Salazar ${ }^{1,2}$ \\ 'Pontifícia Universidade Católica de Goiás - PUC Goiás \\ ${ }^{2}$ Faculdade da Polícia Militar - FPM
}

\section{Resumo}

O3-4 metilenodioximetanfetamina (MDMA) também conhecido popularmente comoecstasyéuma droga sintética comumente usada por frequentadores das chamadas raves. Nãosetem muitos dados epidemiológicos sobre o uso de ecstasy no Brasil, porém o crescimento do número de apreensões da droga no país demonstra que há um aumento no número de usuários. O presente estudo tem o objetivo de descrever o perfil sociodemográfico, econômico e padrão de uso de usuários de ecstasy que moram na cidade de Goiânia-GO. Para realizar essa pesquisa foi realizada uma coleta de dados pormeio da plataforma Google Forms de 60 participantes que se autodeclaram usuários deecstasye moram na cidade de Goiânia-GO. Em relação aos resultados obtidos nesseestudo o perfil dos usuários participantes em sua maioria são jovens, que tem entre 20 e 22 anos (77\%) sendo a maioria do gênero feminino (55\%). Amaiorparteestácursando ensino superior (67\%) ea maioria se classifica como tendo renda de mais de $R \$ 2.862$ a $R \$ 5.724$ (27\%). Quanto ao tempo de uso da droga $45 \%$ dos participantes da pesquisa afirmaram que consomemoecstasyentre 2 e 3 anos. Nesteestudo $98 \%$ dos participantes afirmaram que utilizam o ecstasy em finais de semana e feriados. Dos participantes desta pesquisa, 97\% afirmaram que fazem uso da droga em festas de música eletrônica. Sobre o preço pago pelo comprimido 57\% pagam entre 20 e 30 reais. A droga mais utilizada em associação com o ecstasy foi o álcool seguido pela maconha. A partir dos resultados obtidos pode-se se concluir que é necessário retomar políticas de redução de danos bem como tornar mais efetivas as ações de punição dos produtores e fornecedores da droga e uma fiscalização mais rigorosa da entrada e comercialização da droga em festas.

Palavras-Chave: MDMA, Ecstasy, Drogas sintéticas.

\begin{abstract}
3-4 Methylenedioxymethamphetamine (MDMA) also popularly known as ecstasy is a synthetic drug commonly used by people attending rave parties. There are not many epidemiological data on the use of ecstasy in Brazil, but the growth in the number of drug seizures in the country shows that there is an increase in the number of users. The present study aims to understand the sociodemographic, economicand pattern of use ofecstasy users who live in the city of Goiânia-GO. Toperform this research data were collected through a questionnaire on the Google Forms platform applied to 60 people who declared themselves to be ecstasy users and live in the city of Goiânia-GO. About the results obtained in this study, the profile of the participating users is mostly young, who are between 20 and 22 years old (77\%) and the majorityarefemale (55\%). Mostarestudying at Universities (67\%) and mostareclassified as having an income of more than $R \$ 2,862$ to $R \$ 5,724$ (27\%). Regarding the time of drug use, $45 \%$ of the research participants stated that they had used ecstasy between 2 and 3 years. In this study, 98\% of the participants stated that they use ecstasy on weekends and holidays. Of the participants in this research, 97\% stated that they use the drug at electronic music parties. About the price paid for the
\end{abstract}


tablet, $57 \%$ pay between 20 and 30 reais. The most used drug in association with ecstasy was alcohol followed by marijuana. Based on the results obtained, itcan be concluded that it is necessary to resume damage reduction policies as well as to make the punishment actions of drug producers and suppliers moreeffective and a more rigorous inspection of theentry and commercialization of the drug at parties.

Keywords: MDMA, Ecstasy, Synthetic drug.

\section{Introdução}

O 3-4 metilenodioximetanfetamina (MDMA) também conhecido popularmente como ecstasy, bala, MD ou molly é uma droga sintética comumente usada por frequentadores das chamadas raves, que geralmente são festas de música eletrônica e de longa duração ${ }^{1,2}$.

O MDMA é um derivado anfetamínico, mas que difere das mesmas pela presença de um grupo metilenodioxido ligado a um anel aromático nas posições 3 e 4, que faz com que a estrutura da droga se assemelhe à da mescalina, uma droga alucinógena. Sabe-se também que em função da sua estrutura química o MDMA possui tanto propriedades estimulantes como alucinógenas ${ }^{1,3}$. A figura 1 demonstra a semelhança entre as estruturas químicas do MDMA, mescalina e anfetamina.

Figura 1-Estruturas químicas do MDMA, Mescalina e Anfetamina

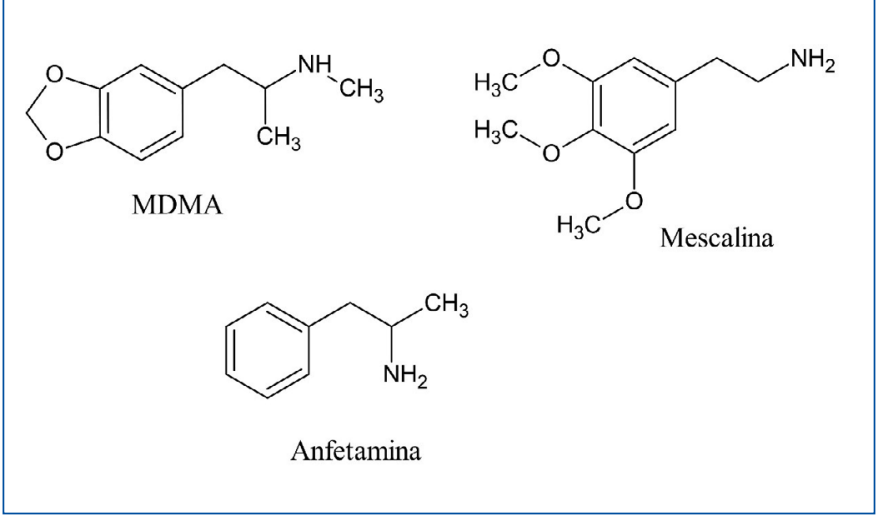

Fonte: Elaborado pelas autoras (2020).

A droga é consumida principalmente por via oral na forma de tabletes, cápsulas, pó e mais comumente comprimidos de diversos formatos e contendo impresso variados logotipos ${ }^{4}$. A concentração de MDMA em cada comprimido varia geralmente de 70 a $100 \mathrm{mg}^{1,3}$. Após a ingestão da droga a ação estimulante do ecstasy é observada em 20 a 60 minutos 5 . A absorção do MDMA ocorre no intestino, e sua concentração sérica máxima é atingida em duas horas. Em um estudo realizado com volun- tários humanos observou-se que doses de 50 mg, 75 mg e 125 mg de MDMA geram uma concentração plasmática de $106 \mathrm{ng} /$ $\mathrm{mL}, 131 \mathrm{ng} / \mathrm{mL}$ e $236 \mathrm{ng} / \mathrm{mL}$. Níveis de toxicidade e até fatalidade foram descritos na literatura com concentrações plasmáticas de MDMA variando entre $0,5 \mathrm{mg} / \mathrm{L}$ a $10 \mathrm{mg} / \mathrm{L}$. Entretanto também houve casos em que a concentração plasmática da droga era baixa $(0,11-0,55 \mathrm{mg} / \mathrm{L})$ demonstrando que a gravidade dos efeitos causados pelo ecstasy pode ser dependente de outros fatores além concentração da substância'. A meia vida do MDMA é de aproximadamente oito horas ${ }^{6}$.

Cerca de $20 \%$ da droga é eliminada pelos rins sem ser metabolizada e o restante após passar pelo fígado é metabolizado pela ação principalmente da enzima CYP2D ${ }^{6}$ (isoenzima do citocromo P450) onde o MDMA é $\mathrm{N}$-desmetilado na substância 3,4-metilenodioxianfetamina (MDA). O MDMA e MDA são O-desmetilados formando 3,4- metilenodiidroxianfetamina (HHA) e o 3,4-diidroxianfetamina (HHMA) que em sequência são O-metilados para formar o 4-hidroxi-3-metoxi-anfetamina (HMA) e o 4-hidroxi-3-metoxi-metanfetamina (HMMA). Esses metabolitos são eliminados na urina ligados ao ácido glicurônico ou sulfato 7,8 .

Com relação ao mecanismo de ação do MDMA, ele atua sobre três neurotransmissores que são: dopamina, serotonina e a noradrenalina induzindo sua liberação e inibindo sua recaptação . Também provoca alterações no eixo hipotálamo-hipófise induzindo a liberação do hormônio adrenocorticotrófico $(\mathrm{ACTH})$, cortisol, ocitocina, arginina e vasopressina ${ }^{10,11}$. Sabe-se que a droga também pode afetar o sistema imune ocasionando em diminuição de linfócitos $C D 4$ no sangue periférico, aumento de células Natural Killer e alteração do perfil de citocinas causando redução na produção de interleucina 2 (IL-2) e interferon gama e aumento a produção de IL-4 e IL-10 ${ }^{12}$.

O MDMA, em geral, causa melhora na comunicação, euforia, aumento da empatia, mudanças de humor e aumento da energia. Alguns autores relatam que são justamente esses efeitos a curto prazo esperados pelos usuários de ecstasy ${ }^{4,13}$.

O consumo da droga, mesmo em doses baixas, também pode gerar sintomas indesejados pelo usuário como: trismo, bruxismo, visão turva, náusea, ansiedade, coordenação motora prejudicada, aumento da sudorese, paranoia, hipertermia, 
tensão muscular, inquietação, taquicardia, entre outros efeitos adversos ${ }^{4,13,14}$.

Após o uso ou até dois dias depois também podem ser observados problemas de concentração, tristeza e cansaço exagerado ${ }^{13}$.

Apesar de a maioria dos efeitos indesejados serem resolvidos em pouco tempo, o consumo de ecstasy pode gerar problemas a longo prazo tanto físicos, quanto psicológicos e neurotoxicidade causada pela liberação excessiva de serotonina demonstrando que a droga não é segura como muitos usuários acreditam'.

Não se tem muitos dados epidemiológicos sobre o uso de ecstasy no Brasil, porém o crescimento do número de apreensões da droga no país demonstra que há um aumento no número de usuários 15. Segundo o Escritório das Nações Unidas sobre Drogas e Crime (UNODC), no Brasil no ano de 2013 foram apreendidos cerca de $50 \mathrm{~kg}$ de ecstasy, já no ano de 2017 o número passou para $205 \mathrm{~kg}^{16}$.

Por outro lado, são poucos os trabalhos que mostram qual é o perfil do usuário de ecstasy no Brasil e nas bases de dados consultadas nenhuma pesquisa nesse sentido foi realizada no Centro-Oeste. Sendo assim, o presente estudo tem o objetivo de descrever o perfil sociodemográfico, econômico e padrão de uso de usuários de ecstasy residentes na cidade de Goiânia, Goiás.

\section{Métodos}

A pesquisa trata-se de um estudo descritivo, quantitativo, que tem por finalidade analisar o perfil socioeconômico, demográfico e padrão de uso dos jovens maiores de idade usuários de ecstasy na cidade de Goiânia-GO.

Antes de realizar a coleta de dados, o projeto foi aprovado pelo Comitê de Ética em Pesquisa da PUC-Goiás com número de parecer: 4.077.524.

A pesquisa contou com 60 participantes maiores de idade (acima de 18 anos), residentes na cidade de Goiânia e que se autodeclaram abertamente usuários de ecstasy.

O procedimento de coleta de dados durou de Julho a Agosto de 2020 e foi realizado por meio de um formulário desenvolvido pelas pesquisadoras e aplicado na plataforma Google Forms. $O$ formulário era composto por nove questões de múltipla escolha com indagações sociodemográficas e econômicas sobre os participantes e específicas sobre o uso do ecstasy.

O link para acesso ao questionário foi enviado por mensagem através da rede social particular acompanhado de um texto convidando individualmente a participar da pesquisa. Os indivíduos que tiveram interesse em participar da pesquisa só tiveram acesso às perguntas após concordarem com o Termo de Consentimento Livre e Esclarecido presente no início do formulário.
Para tabulação dos dados obtidos através dos questionários foi utilizado o programa Microsoft Office Excel ${ }^{\circledR}$ versão 2002.

\section{Resultados e Discussão}

O MDMA é uma droga sintética que apresenta propriedades estimulantes e alucinógenas, amplamente utilizada por jovens adultos e até menores de idade, principalmente em festas de música eletrônica. Com relação ao perfil sociodemográfico e econômico obtido os resultados estão contidos na tabela 1 .

Tabela 1 -Dados sobre o perfil sociodemográficoe socioeconômico dos indivíduos autodeclarados usuários de ecstasy que moram em Goiânia-GO (N=60).

\begin{tabular}{|c|c|c|c|}
\hline Parâmetro pesquisado & & $\mathbf{N}$ & $\%$ \\
\hline \multirow[t]{2}{*}{ Gênero de identificação } & Feminino & 33 & 55 \\
\hline & Masculino & 27 & 45 \\
\hline \multirow[t]{10}{*}{ Idade } & 18 & 2 & 3 \\
\hline & 19 & 2 & 3 \\
\hline & 20 & 12 & 20 \\
\hline & 21 & 19 & 32 \\
\hline & 22 & 15 & 25 \\
\hline & 23 & 5 & 8 \\
\hline & 24 & 1 & 2 \\
\hline & 25 & 1 & 2 \\
\hline & 26 & 2 & 3 \\
\hline & 27 & 1 & 2 \\
\hline \multirow[t]{4}{*}{ Grau de escolaridade } & Ensino médio completo & 5 & 8 \\
\hline & Cursando Ensino superior & 40 & 67 \\
\hline & $\begin{array}{l}\text { Ensino Superior in- } \\
\text { completo (matrícula } \\
\text { trancada) }\end{array}$ & 10 & 17 \\
\hline & Ensino superior completo & 5 & 8 \\
\hline \multirow[t]{7}{*}{ Grupo socioeconômico* } & Grupo 1 & 4 & 7 \\
\hline & Grupo 2 & 5 & 8 \\
\hline & Grupo 3 & 16 & 27 \\
\hline & Grupo 4 & 11 & 18 \\
\hline & Grupo 5 & 10 & 17 \\
\hline & Grupo 6 & 8 & 10 \\
\hline & Grupo 7 & 6 & 13 \\
\hline
\end{tabular}

* Para analisar o grupo socioeconômico foi utilizada uma tabela do Instituto Brasileiro de Geografia e Estatística (IBGE) (2017) que classifica os indivíduos em 7 grupos distintos de acordo com a renda familiar (somatório da renda individual dos moradores do mesmo domicílio). $\mathrm{O}$ grupo 1 tem renda 
mensal de até $R \$ 1908$, o grupo 2 tem renda mensal de mais de $R \$ 1908$ a $R \$ 2.862$, o grupo 3 tem renda de mais de $R \$ 2.862$ a $R \$ 5.724$. o grupo 4 de mais de $R \$ 5.724$ a $R \$ 9.540$, o grupo 5 de mais $R \$ 9.540$ a $R \$ 14.310$, grupo 6 de mais de 14.310 a $R \$ 23.850$ e grupo 7 mais de $R \$ 23.850^{17}$.

Em relação aos resultados obtidos nesse estudo, o perfil dos usuários participantes são jovens, que tem entre 20 e 22 anos (77\%), sendo a maioria do gênero feminino (55\%). A maior parte está cursando ensino superior (67\%) e a maioria dos participantes se classifica como pertencentes do grupo socioeconômico 3 (27\%). Esses resultados são semelhantes aos encontrados nas pesquisas realizadas por Almeida \& Silva (2003)18 e Battisti e colaboradores (2006)19 na cidade de São Paulo nas quais entrevistaram 52 e 32 usuários respectivamente. Esses autores obtiveram que o padrão de usuários da mesma droga em São Paulo são jovens adultos, do sexo masculino, heterossexuais e com alto grau de escolaridade (ensino superior completo ou incompleto). Embora os resultados tenham sido similares aos encontrados no presente trabalho, houve uma variação no gênero entre os entrevistados. No estudo de Almeida e Silva e Battisti e colaboradores a maioria dos participantes foram do gênero masculino, já no presente trabalho a maioria foram pessoas do gênero feminino. $\mathrm{O}$ aumento do consumo de drogas por mulheres pode ser consequência das mudanças ocorridas no papel social da mulher, como a maior inserção no mercado de trabalho, porém ainda sendo subvalorizadas e recebendo salários menores quando comparado a pessoas do gênero masculino, logo o uso de drogas pode ser uma forma de lidar com problemas do cotidiano ${ }^{20,21}$.

Sobre o nível socioeconômico os resultados obtidos neste estudo corroboram que o que foi encontrado por Batistti e colaboradores (2006)19 onde 15 participantes do seu estudo se auto classificaram como sendo nível médio e 11 como nível médio alto e com os resultados de Almeida e Silva (2003) 18 onde $53 \%$ dos participantes se classificaram como sendo de nível socioeconômico médio. A porcentagem de pessoas encontradas nessa pesquisa que são dos grupos 1, 2 e 3 (42\%) demonstra que a droga não está associada apenas a usuários de níveis econômicos mais altos.

O ecstasy é consumido principalmente via oral geralmente na forma de comprimidos. A quantidade de comprimidos utilizada varia entre dois a três por noite podendo chegar a até 25 comprimidos pelos usuários mais experientes ${ }^{22,23}$, o que significaria um alerta importante para os possíveis casos de intoxicação ou até morte após o consumo da droga. A tabela 2 demonstra os resultados sobre o padrão de uso do ecstasy obtidos no presente trabalho.
Tabela 2 - Dados sobre o padrão de uso de ecstasy dos indivíduos que se auto declaram usuários e que moram na cidade de Goiânia$\mathrm{GO}(\mathrm{N}=60)$.

\begin{tabular}{|c|c|c|c|}
\hline Parâmetro pesquisado & & $\mathbf{N}$ & $\%$ \\
\hline \multirow[t]{4}{*}{ Tempo de consumo } & Menos de 1 ano & 2 & 3 \\
\hline & Entre 1 e 2 anos & 10 & 17 \\
\hline & Entre 2 e 3 anos & 27 & 45 \\
\hline & Mais de 4 anos & 21 & 35 \\
\hline \multirow[t]{2}{*}{ Tempo de consumo } & Finais de semana e feriados & 59 & 98 \\
\hline & Qualquer dia da semana & 1 & 2 \\
\hline \multirow[t]{7}{*}{ Locais de uso } & $\begin{array}{l}\text { Em festas de música } \\
\text { eletrônica }\end{array}$ & 58 & 97 \\
\hline & $\begin{array}{l}\text { Em festas de outros } \\
\text { gêneros musicais (funk, } \\
\text { sertanejo, pagode, etc.) }\end{array}$ & 28 & 47 \\
\hline & Em Boates & 28 & 47 \\
\hline & $\begin{array}{l}\text { Em reuniões na casa de } \\
\text { amigos }\end{array}$ & 28 & 47 \\
\hline & Em Festivais de música & 53 & 88 \\
\hline & Em Bares & 8 & 13 \\
\hline & Em casa & 8 & 13 \\
\hline \multirow[t]{4}{*}{$\begin{array}{l}\text { Preço pago pelo } \\
\text { comprimido de ecstasy }\end{array}$} & Menos de 20 reais & 18 & 30 \\
\hline & Entre 20 e 30 reais & 34 & 57 \\
\hline & Entre 30 e 40 reais & 3 & 5 \\
\hline & Mais de 40 reais & 5 & 8 \\
\hline \multirow[t]{7}{*}{$\begin{array}{l}\text { Uso de outras drogas } \\
\text { associadas ao ecstasy }\end{array}$} & Não & 2 & 3 \\
\hline & Álcool & 53 & 88 \\
\hline & Tabaco & 47 & 70 \\
\hline & Maconha & 44 & 73 \\
\hline & Cocaína & 8 & 13 \\
\hline & $\begin{array}{l}\text { Inalante (loló ou lança } \\
\text { perfume) }\end{array}$ & 17 & 28 \\
\hline & LSD (papel) & 21 & 35 \\
\hline
\end{tabular}

Quanto ao tempo de uso da droga 45\% ( $n=27)$ dos participantes da pesquisa afirmaram que consomem o ecstasy entre 2 e 3 anos, 35\% ( $n=21)$ a mais de 4 anos, $17 \%(n=10)$ entre 1 e 2 anos e $3 \%(n=2)$ a menos de 1 ano. Comparando os tempos de uso prevalentes de ecstasy que foram entre 2 e 3 anos e de 4 anos ou mais com a idade da maioria dos participantes (20 a 22 anos) assume-se que alguns deles começaram a consumir a droga quando eram menores de idade. Almeida e Silva (2003)18 obtiveram em seu estudo que $23,5 \%$ dos participantes experimentaram a droga ainda menores de idade. Esse fato pode ser consequência da facilidade em que se têm de adquirir a sub- 
stância. Com relação a facilidade para obtenção da droga, em um estudo realizado em Atlanta, Georgia entre os anos 2002 a 2007 por Bahora e colaboradores ${ }^{24}$ onde entrevistaram 112 usuários de ecstasy, um participante afirmou que adquirir a droga é tão fácil quanto comprar um pacote de batatas fritas. Essa facilidade de acesso à droga foi corroborada no trabalho de Almeida e Silva (2003) ${ }^{18}$ realizado em São Paulo onde eles obtiveram que a maioria das pessoas não compra a droga de traficantes, mas sim de algum amigo ou conhecido.

Outro fator a ser considerado é a inserção de meios digitais para comercialização do ecstasy como o Criptomercado na Dark Web que usa de artifícios como a criptografia e o pagamento por meio moedas digitais e permite ao vendedor e o comprador permanecerem em anônimo. Com o advento do uso de Smartphones, as redes sociais como Whatsapp, Snapchat, Instagram também passaram a ser um canal que possibilita a venda de drogas e permite ao usuário uma maior facilidade, comodidade e segurança para adquirir a substância ${ }^{25,26}$.

Sobre a frequência de uso, neste estudo 59 (98\%) participantes afirmaram que utilizam o ecstasy em finais de semana e feriados, um participante afirmou que usa a droga qualquer dia da semana, corroborando com o que foi encontrado por Bahora e colaboradores (2009) ${ }^{24}$ em sua pesquisa realizada por meio de entrevistas, onde grande parte dos participantes afirmaram que utilizam a droga para fins recreativos como em finais de semana, feriados ou ocasiões especiais e na companhia de amigos ou membros da família.

Dos participantes desta pesquisa, $97 \%(n=58)$ afirmaram que fazem uso da droga em festas de música eletrônica, $88 \%$ utilizam em festivais de música, $47 \%$ usam a droga em reuniões em casas de amigos, $47 \%$ utilizam em boates, $47 \%$ utilizam em festas de outros gêneros musicais (funk, sertanejo, pagode etc.), $13 \%$ utilizam em bares e $13 \%$ em casa. Almeida \& Silva (2003) 18 também verificaram em seu estudo que o principal lugar onde a droga é utilizada são em festas de música eletrônica (78\%) seguido de boates (69,2\%) e festas de outros gêneros (53,8\%). A preferência pelo uso da droga em festas raves (festas de música eletrônica e de longa duração) $)^{1,2}$ pode ser devido aos efeitos causados pelo ecstasy, que são aumento da resistência física, diminuição do sono e cansaço, sensação de bem-estar, aumento a sociabilidade e sensação de felicidade ${ }^{4,13}$.

De acordo com os resultados obtidos no presente estudo, o preço pago pelo comprimido de ecstasy variou entre menos de 20 reais até mais de 40 reais. Como demonstrado na tabela 2 , dos participantes da pesquisa $57 \%(n=34)$ pagam entre $20 \mathrm{e}$ 30 reais, $30 \%(n=18)$ afirmaram que pagam menos de 20 reais no comprimido de ecstasy, $8 \%(n=5)$ pagam mais de 40 reais e $5 \%(n=3)$ pagam entre 30 e 40 reais. Este dado é semelhante ao obtido por Battisti e colaboradores $(2006){ }^{19}$ que observaram em seu estudo que o preço pago pela droga variou de 25 a 50 reais. A diferença de valores pagos pela droga em diferentes regiões do Brasil ou até dentro da mesma cidade, pode ser ocasionada pela diferença da composição dos comprimidos. Diversos trabalhos demostram que os comprimidos vendidos como ecstasy podem conter apenas o MDMA ou substâncias similares como metilenodioxietilanfetamina (MDEA), 3,4-metilenodioxianfetamina (MDA), metanfetamina e anfetamina e podem ser encontrados outros adulterantes como a cafeína e a efedrina ${ }^{4,27-29}$. Por outro lado, como os comprimidos não passam por nenhuma fiscalização toxicológica, não há como confirmar que o de maior valor tenha maior grau de pureza, logo os usuários estão sujeitos aos efeitos adversos que podem ser causados pelas substâncias adulterantes.

Com relação ao uso concomitante de mais de uma droga, como pode ser visto na tabela 2 , a droga mais utilizada em associação com o ecstasy foi o álcool ( $88 \%$ dos participantes) seguida pela maconha (73\% dos participantes). Battisti e colaboradores (2006)19 em seu estudo realizado na cidade de São Paulo com 32 participantes verificaram que a droga mais usada conjuntamente com ecstasy foi a maconha e em segundo lugar o álcool. Já na pesquisa realizada por Almeida \& Silva (2003) ${ }^{18}$ também na cidade de São Paulo, onde participaram 52 pessoas observou-se que a principal droga utilizada com o ecstasy foi a maconha seguida pelo tabaco sendo o álcool a quarta droga mais consumida em associação. Oálcool diferente da maconha, é uma droga mais acessível, pois é lícita e pode ser comercializada legalmente nos eventos e estabelecimentos podendo essa ser uma explicação para a maior prevalência do seu consumo associado ao ecstasy. O uso do álcool associado ao ecstasy leva os usuários a consumirem maior quantidade de etanol sem que sintam os efeitos imediatos causados pela substância. A utilização de ambas as drogas também está relacionada com um maior comportamento de risco por parte dos usuários como sexo sem proteção e condução de automóveis sobre efeito das drogas30. Além disso, em um estudo randomizado duplo-cego realizado por Hernández-López e colaboradores (2002) onde nove voluntários do sexo masculino consumiram $100 \mathrm{mg}$ de ecstasy e $0,8 \mathrm{~g}$ de etanol $/ \mathrm{kg}$ ou $100 \mathrm{mg}$ de ecstasy e $0,8 \mathrm{~g}$ de bebida placebo/kg foi demonstrado que o álcool utilizado com o ecstasy pode causar aumento das concentrações plasmáticas de MDMA em até $13 \%{ }^{31}$. O efeito cumulativo do ecstasy pode ocasionar sequelas aos usuários pois o MDMA tem grande potencial tóxico.

A intoxicação aguda causada pelo ecstasy pode ocasionar complicações orgânicas e levar até a morte ${ }^{32}$. Um exemplo disso é a hipertermia gerada pela droga que pode levar ao de- 
senvolvimento de complicações sistêmicas como coagulação intravascular disseminada (CIVD), rabdomiólise e falência múltipla de órgãos ${ }^{6,14}$. Calle e colaboradores $(2019)^{33}$ realizaram uma pesquisa em duas edições de um evento de música eletrônica a céu aberto que aconteceram nos anos de 2013 e 2014 onde participaram 30.000 e 26.000 pessoas respectivamente. Cerca de 160 pessoas procuraram atendimento no posto médico do evento com sintomas de intoxicação por drogas. $\mathrm{Na}$ análise de sangue realizada em 106 pacientes intoxicados, 36 amostras detectaram a presença de MDMA, porém em apenas em seis amostras o ecstasy era a única droga presente, nas outras 30 amostras havia a presença de etanol (29 amostras), Tetrahidrocanabinol (THC) (11 amostras), cocaína (seis amostras) e anfetamina (duas amostras) demonstrando que o poli uso de drogas é uma tendência entre os usuários de ecstasy mas que pode acarretar intoxicação.

Foi demonstrado através de pesquisas que usuários de ecstasy tem notas acadêmicas mais baixas que não usuários. Também foi observado que os usuários da droga têm mais problemas familiares, de relacionamento, ocupacionais e financeiros se comparado a pessoas que não fazem uso do ecstasy, demonstrando que a droga pode causar problemas sociais ao usuário além dos problemas físicos e psicológicos relacionados com a droga ${ }^{34}$.

\section{Conclusão}

Pode-se concluir a partir dos resultados obtidos no presente estudo que os usuários de ecstasy participantes da presente pesquisa são jovens adultos, sendo a maioria do gênero feminino, tem boa escolaridade, são de nível socioeconômico médio e alto, associam ecstasy com outras drogas, principalmente álcool e tem fácil acesso as mesmas e em alguns casos começaram a consumir o ecstasy quando ainda eram menores de idade.

Portanto torna-se de extrema importância retomar políticas de redução de danos estimulando a conscientização de adolescentes e jovens ao respeito dos riscos aos quais estão sendo submetidos, bem como tornar mais efetivas as ações de punição dos produtores e fornecedores da droga e uma fiscalização mais rigorosa da entrada e comercialização da droga em festas.

\section{Referências}

1. Kalant H.The pharmacology and toxicology of "ecstasy" (MDMA) and related drugs. CMAJ. 2001;165(7):917-928.

2. Campos Baptista M, Noto AR, Nappo S, De Araújo Carlini E. O uso de êxtase (MDMA) na cidade de São Paulo e imediações: Um estudo etnográfico. J Bras Psiquiatr. 2002;51:81-89.

3. Sardinha LS, Garcia AL. Um estudo sobre a psi- codinâmica do usuário de ecstasy. Psicol Teor e Prática. 2000;2(2):70-87.

4. Siegel RK. MDMA: Nonmedical use and intoxication. J Psychoactive Drugs. 1986;18(4):349-354.

5. Laranjeira RR, Dunn J, Rassi R, Fernandes M. 'Extase (3,4 metilenodioximetanfetamina, MDMA): Uma droga velha e um problema novo? Rev da Assoc Bras Psiquiatr. 1996;18(3):77-81.

6. Ferigolo M, Machado AG d. S, Oliveira NB, Barros HMT.Ecstasy intoxication: the toxicological basis for treatment. Rev Hosp Clin Fac Med Sao Paulo. 2003;58(6):332-41.

7. Wu D, Victoria Otton $S$, Inaba T, Kalow W, Sellers EM. Interactions of amphetamine analogs with human liver CYP2D6. Biochem Pharmacol. 1997;53(11):1605-12.

8. Schifano F. A bitter pill. Overview of ecstasy (MDMA, MDA) related fatalities. Psychopharmacology. 2004;173(3-4):242-248.

9. Green AR, Cross AJ, Goodwin GM. Review of the pharmacology and clinical pharmacology of 3,4-methylenedioxymethamphetamine (MDMA or "Ecstasy"). Psychopharmacology. 1995;119(3):247-260.

10. Grob CS, Poland RE, Chang L, Ernst T. Psychobiologic effects of 3,4-methylenedioxymethamphetamine in humans: methodological considerations and preliminary observations. Behav Brain Res. 1996;73(1-2):103-107.

11. Henry JA, Fallon JK, Kicman AT, Hutt AJ, Cowan DA, Forsling M. Low-dose MDMA ('ecstasy') induces vasopressin secretion. Lancet. 1998;351(9118):1784.

12. Pacifici R, Zuccaro P, Farré M, Pichini S, Di Carlo S, Roset $\mathrm{PN}$, et al. Effects of repeated doses of MDMA ("Ecstasy") on cell-mediated immune response in humans. Life Sci. 2001;69(24):2931-2941.

13. 13. Cohen RS. Subjective reports on the effects of the MDMA ('ecstasy') experience in humans. Prog Neuropsychopharmacol Biol Psychiatry. 1995;19(7):1137-1145.

14. Henry JA, Jeffreys KJ, Dawling S. Toxicity and deaths from 3,4-methylenedioxymethamphetamine ("ecstasy"). Lancet. 1992;340(8816):384-387.

15. United Nations Office on Drugs and Crime (UNODC). Relatório Mundial Sobre Drogas 2008 [acesso em: 02 out 2020]. Disponível em : http://www.unodc.org/unodc/ en/data-and-analysis/WDR-2008.html

16. United Nations Office on Drugs and Crime (UNODC). Relatório Mundial Sobre Drogas 2019; [acesso em:02 out 2020]. Disponível em: https://wdr.unodc.org/wdr2019/ prelaunch/WDR19_Booklet_4_STIMULANTS.pdf.

17. Instituto Brasileiro de Geografia e Estatistica I. Pesquisa de Orçamentos Familiares 2017 - 2018 - Primeiros Re- 
sultados [acesso em: 05 de mar 2020]. Disponível em: http://scholar.google.com/scholar?hl=en\&btnG=Sear ch\&q=intitle:Pesquisa+de+Or?amentos+Familiares\#0

18. Almeida SP de, Silva MTA. Ecstasy (MDMA): effects and patterns of use reported by users in São Paulo. Rev Bras Psiquiatr. 2003;25(1):11-17.

19. Battisti MC, Noto AR, Nappo S, Carlini EDA. A Profile of ecstasy (MDMA) use in Sāo Paulo, Brazil: An ethnographic study. J Psychoactive Drugs. 2006;38(1):13-18.

20. Romo N. Género Y Uso De Drogas: la invisibilidad de las mujeres. Monografías Humanitas. 2006;5:65-83.

21. Marangoni SR, de Oliveira MLF. Triggering factors for drug abuse in women. Texto e Context Enferm. 2013;22(3):662-670.

22. Burgess C, O'Donohoe A, Gill M. Agony and ecstasy: A review of MDMA effects and toxicity. Eur Psychiatry. 2000;15(5):287-294.

23. Gouzoulis-Mayfrank E, Daumann J. The confounding problem of polydrug use in recreational ecstasy/ MDMA users: A brief overview. J Psychopharmacol. 2006;20(2):188-193.

24. Bahora M, Sterk CE, Elifson KW. Understanding recreational ecstasy use in the United States: A qualitative inquiry. Int J Drug Policy. 2009;20(1):62-69.

25. Demant J, Bakken SA, Oksanen A, Gunnlaugsson H. Drug dealing on Facebook, Snapchat and Instagram: A qualitative analysis of novel drug markets in the Nordic countries. Drug Alcohol Rev. 2019;38(4):377-385.

26. Moyle L, Childs A, Coomber R, Barratt MJ. \#Drugsforsale: An exploration of the use of social media and encrypted messaging apps to supply and access drugs. Int J Drug Policy.2019;63:101-110.

27. Lapachinske SF, Yonamine M, Moreau RL de M. Validação de método para determinação de 3,4-metilenodioximetanfetamina (MDMA) em comprimidos de ecstasy por cromatografia em fase gasosa. Rev Bras Ciências Farm. 2004;40(1):75-83.

28. Teng SF, Wu SC, Liu C, Li JH, Chien CS. Characteristics and trends of 3,4-methylenedioxymethamphetamine (MDMA) tablets found in Taiwan from 2002 to February 2005. Forensic Sci Int. 2006;161(2-3):202-208.

29. Giraudon I, Bello PY. Monitoring ecstasy content in France: Results from the National Surveillance System 1999-2004. Subst Use Misuse. 2007;42(10):1567-1578.

30. Breen $C$, Degenhardt $L$, Kinner $S$, Bruno R, Jenkinson $R$, Matthews $A$, et al. Alcohol use and risk taking among regular ecstasy users. Subst Use Misuse. 2006;41(8):1095-1109.
31. Hernández-López $C$, Farré $M$, Roset PN, Menoyo $E$, Pizarro N, Ortuño J, et al. 3,4-Methylenedioxymethamphetamine (ecstasy) and alcohol interactions in humans: Psychomotor performance, subjective effects, and pharmacokinetics. J Pharmacol Exp Ther. 2002;300(1):236-244.

32. Brugada P, Brugada J. Right bundle branch block, persistent ST segment elevation and sudden cardiac death: A distinct clinical and electrocardiographic syndrome. A multicenter report. J Am Coll Cardiol. 1992;20(6):13911396.

33. Calle $P$, Sundahl N, Maudens $K$, Wille SM, Van Sassenbroeck D, De Graeve K, et al. Medical Emergencies Related to Ethanol and Illicit Drugs at an Annual, Nocturnal, Indoor, Electronic Dance Music Event. Prehosp Disaster Med. 2018;33(1):71-76.

34. Singer LT, Linares TJ, Ntiri S, Henry R, Minnes S. Psychosocial profiles of older adolescent MDMA users. Drug Alcohol Depend. 2004;74(3):245-52. 